\title{
Profound Hypoxemia From Right Ventricular Failure Following Acute Type A Aortic Dissection
}

\author{
Shi Hui Tan, b, Cheryl Chun Lei Tan ${ }^{\mathrm{a}}$, Yi Lin Lee
}

\begin{abstract}
Acute aortic dissection is a catastrophic event with high mortality rate if left untreated. Complications of aortic dissection are fairly common, and some of them increase mortality rates further, necessitating early diagnosis and treatment. We present a case of Stanford type A aortic dissection with an uncommon complication of right ventricular failure, which resulted in a rare presentation of persistent hypoxemia despite intubation and maximal ventilatory support. Other common causes of hypoxemia were ruled out and this was eventually attributed to the aortic dissection and emergency surgery was arranged for the patient. Our case can help to increase the awareness of such a potential association, which should be considered in future similar clinical situations, thus minimizing any delay in management.
\end{abstract}

Keywords: Aortic dissection; Right ventricular failure; Hypoxemia

\section{Introduction}

Acute aortic dissection is the most common surgical emergency among all aortic conditions and can be classified into Stanford type A which involves the ascending aorta, and type $\mathrm{B}$ which occurs distal to the left subclavian artery. In particular, acute Stanford type A aortic dissection has a mortality rate that reaches $50 \%$ by the first $48 \mathrm{~h}$ if left untreated [1]. Prompt diagnosis and surgical treatment can improve patient outcomes. We report a case of a relatively well patient with acute Stanford type A aortic dissection with a rare complication of right heart failure who presented with persistent profound hypoxemia despite intubation, and discuss the possible etiologies.

Manuscript submitted January 14, 2021, accepted January 21, 2021

Published online February 8, 2021

aDepartment of Anaesthesiology, Singapore General Hospital, Singapore ${ }^{b}$ Corresponding Author: Shi Hui Tan, Department of Anaesthesiology, Singapore General Hospital, Outram Road, Singapore 169608, Singapore.

Email: tan.shi.hui@singhealth.com.sg

doi: https://doi.org/10.14740/jmc3652

\section{Case Report}

\section{Investigations}

A 70-year-old lady with well-controlled hypertension and multinodular goiter status post total thyroidectomy presented to our emergency department with a sudden onset of chest pain radiating to bilateral arms, shortness of breath and diaphoresis. On examination, her blood pressure was $84 / 30 \mathrm{~mm}$ $\mathrm{Hg}$, heart rate was $47 / \mathrm{min}$ and oxygen saturation was $82 \%$ on room air. The patient was alert and responsive at the time of presentation, and cardiovascular examination did not reveal any murmurs or signs suggestive of heart failure such as pedal edema or lung crepitations. Her neurological examination was also grossly normal. Intravenous dopamine infusion up to $20 \mu \mathrm{g} / \mathrm{kg} / \mathrm{min}$ was immediately started to support her hemodynamics and she was given supplemental oxygen. She was diagnosed to have an acute inferior ST elevation myocardial infarction (STEMI) based on electrocardiogram showing ST elevation in inferior leads and a complete heart block (CHB), and was sent for urgent primary percutaneous coronary intervention.

\section{Diagnosis}

Coronary angiograpy revealed normal left coronary artery, a dissection flap in the aortic root arising from the right coronary cusp and the right coronary artery (RCA) ostium was unable to be located, likely occluded by the dissection flap. Bedside echocardiogram done showed ascending aortic dissection with dilated aortic root and mild aortic regurgitation. She was intubated in the catheterization laboratory in view of persistent hypoxemia. Despite intubation and hand ventilation with $100 \%$ oxygen, she remained hypoxic with oxygen saturation of $80 \%$ and a corresponding arterial blood gas showed a partial pressure of oxygen $\left(\mathrm{PaO}_{2}\right)$ of $41 \mathrm{~mm} \mathrm{Hg}$. Endotracheal tube (ETT) placement was confirmed with 5-point auscultation, repeat laryngoscopies as well as chest X-ray (CXR). No pneumothoraxes were seen on the CXR. She was started on IV noradrenaline infusion up to 0.4 $\mu \mathrm{g} / \mathrm{kg} / \mathrm{min}$ and transvenous pacing for the CHB.

In view of her persistent hypoxemia, computed tomography (CT) aortogram and thorax were performed. The CT aortogram showed a Stanford type A aortic dissection extending to the right common and left external iliac arteries with RCA arising from the false lumen with poor contrast opacification. 


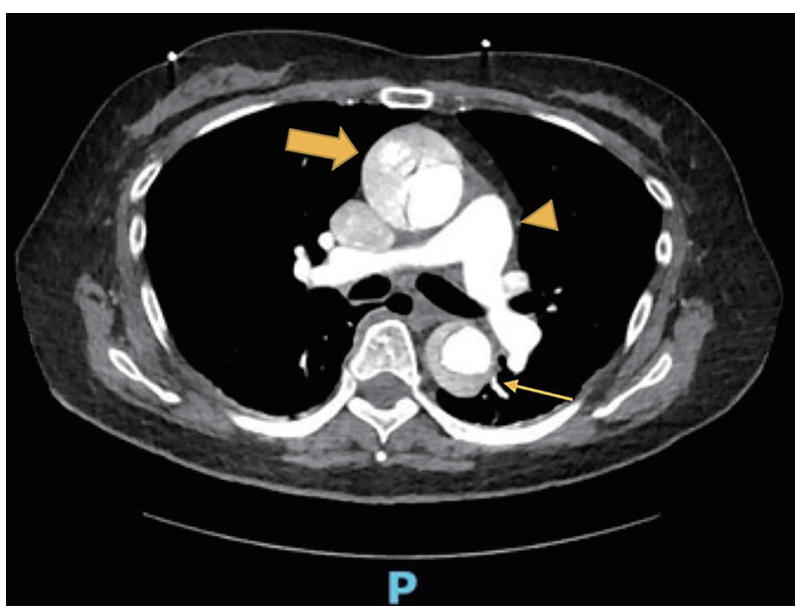

Figure 1. Computed tomography (CT) angiogram showing aortic dissection in the ascending aorta (arrow) and descending aorta (thin arrow), and no clots within the pulmonary artery to suggest pulmonary embolism (arrowhead).

There was no evidence of pulmonary embolism or lung consolidation (Fig. 1).

\section{Treatment}

The patient was transferred to the operating theater for a coronary artery bypass graft of the RCA using saphenous vein graft and a repair of the dissection. Intra-operatively, the right ventricle was observed to be infarcted with akinesia, and no flow was seen in the RCA. Despite these surgical interventions, poor right ventricular function with minimal ejection into the pulmonary artery was noted, and decision was made to commence veno-arterial extracorporeal membrane oxygenation (ECMO) as a bridge to recovery.

\section{Follow-up and outcomes}

Post-operatively, her recovery was complicated by acute kidney injury and hemorrhagic shock. Despite extensive resuscitation, there was persistently low flow on the ECMO and her pupils were noted to be dilated with sluggish response to light on post-operative day 1. CT of brain was performed which showed hypoxic-ischemic encephalopathy. Poor prognosis for meaningful survival was conveyed to the family, and a decision was made for no further escalation of care. ECMO was also subsequently withdrawn and the patient eventually passed away on post-operative day 3 .

\section{Discussion}

Aortic dissection is relatively uncommon, with an incidence of two to six cases per 100,000 persons [2], but it is associated with high morbidity and mortality rates. Aortic dissection is characterized by the separation of the intima-media layers within the aortic wall and the extent and direction of dissection determines the patients' presentation. Classically, patients may experience an abrupt onset of tearing/ripping chest or back pain, but it can also mimic other medical conditions such as myocardial ischemia, heart failure, cerebrovascular accident and pulmonary embolism [3]. Evangelista et al reported that over a span of 20 years, medical mortality rate in patients with type A aortic dissection remains high at $57 \%$, while surgical mortality rate has declined, highlighting the importance of early surgical intervention in the treatment of these patients [3].

Complications from aortic dissection can occur rapidly, and include shock (15\%), cardiac tamponade (18\%), mesenteric malperfusion (3.7\%) and brain injury (10\%) [3]. These complications arise due to the dissection affecting mesenteric and arch vessels respectively. Congestive heart failure has an incidence of $6 \%$ [4], while right ventricular failure (RVF) has only been reported in case series $[5,6]$. Such complications significantly increase mortality rate, and should be elucidated early when assessing patients.

Unfortunately, despite being a catastrophic condition, aortic dissection is often misdiagnosed resulting in delayed treatment and a negative patient outcome. The myriad of presentations, either as a direct effect of the acute aortic dissection or a complication of it, can mislead the medical practitioner. Our patient was initially misdiagnosed with an inferior STEMI and a CHB and was brought for urgent coronary revascularization. It was only during the procedure that she was noted to have an aortic dissection. Acute STEMI can occur due to the occlusion of the flow within the coronary arteries by the dissection flap if the dissection extends proximally. However, what remains unusual is that she remained profoundly hypoxemia, even after intubation and $100 \%$ oxygen delivered, with a $\mathrm{PaO}_{2} /$ fraction of inspiration $\mathrm{O}_{2}\left(\mathrm{FiO}_{2}\right)$ ratio of 41 and normal lung parenchyma. This is likely due to RVF from the aortic dissection extension to the RCA, leading to right ventricular infarction and akinesia. There is a resultant forward failure with the inability to pump blood into the pulmonary circulation for oxygenation to occur. The additional presence of intra-cardiac shunts can further worsen hypoxemia as it allows the mixture of oxygenated and deoxygenated blood. Even though such shunts were not detected in our patient, small shunts could be easily overlooked if these were not intentionally sought after during the echocardiogram. Similar mechanisms in patients with RVF and infarction have previously only been described in case reports $[7,8]$. To the best of our knowledge, such profound hypoxemia in aortic dissection and its proposed mechanism have not been reported in the literature to date.

RVF is often overlooked in the discussion of heart failure. The causes of RVF include cardiac conditions such as left ventricular failure (LVF), direct right ventricular myocardial disease such as right ventricular ischemia or infarction, or constrictive pericarditis and lung pathologies like pulmonary embolism, pulmonary hypertension [9]. Symptoms include dyspnea, light-headedness, syncope, right upper quadrant discomfort and peripheral edema, while physical examination findings include systemic hypotension, tachycardia, elevated jugular venous pressure, right ventricular third sound and tricuspid regurgitation [10]. RVF is easily missed due to the nonspecific nature of these clinical presentations, and clinicians 
Table 1. Causes of Hypoxemia Persisting Despite Intubation

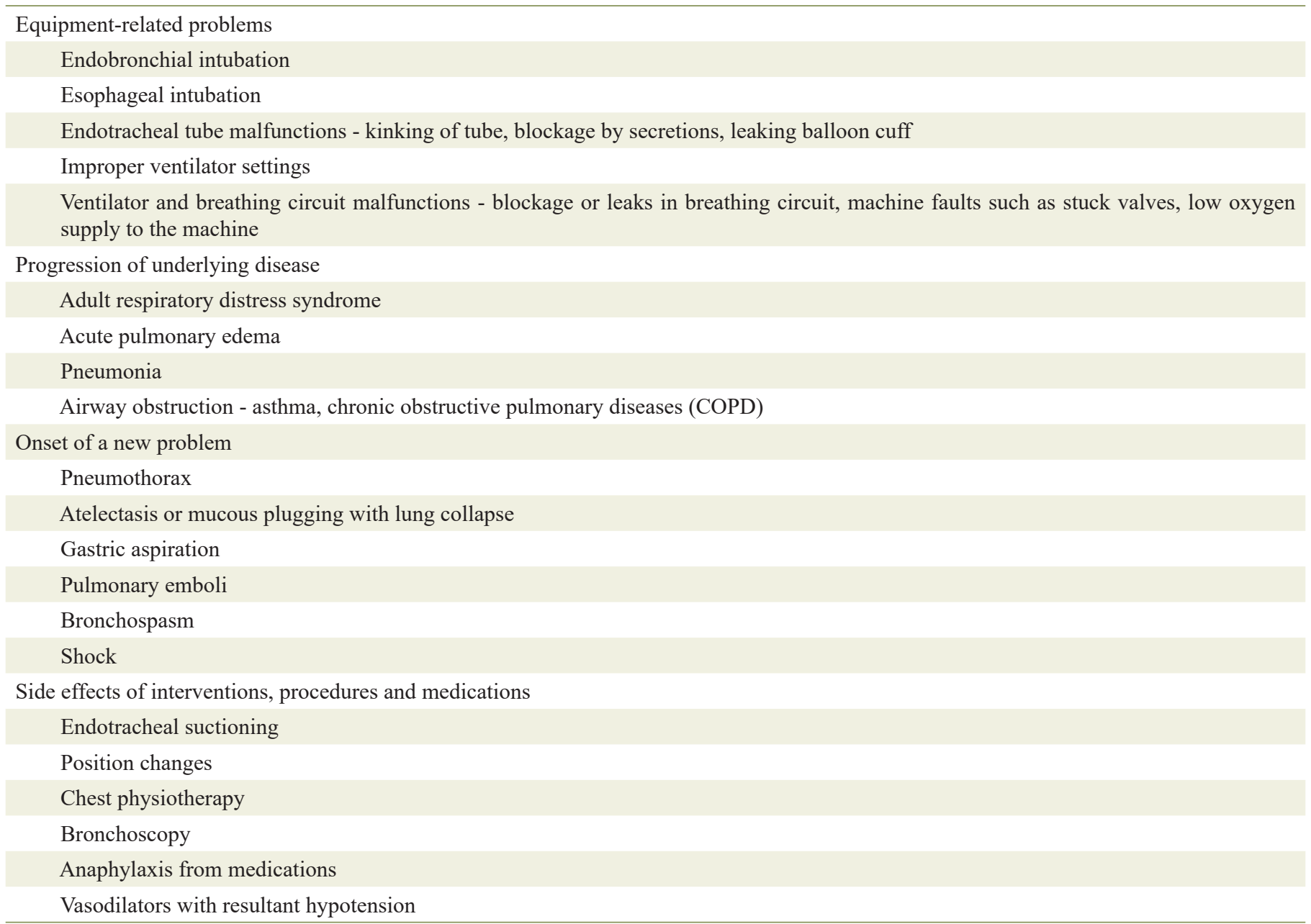

need to maintain a high index of suspicion to clinch this diagnosis. While RVF can cause forward failure through interventricular dependence, compromising LV preload and reducing LV performance, resulting in hypotension and hypoperfusion [11], there is a paucity of literature demonstrating hypoxia as a direct consequence of RVF. RVF reversibility is more likely and rapid than LVF due to the thinner nature of the RV [11], hence prompt diagnosis and early institution of treatment is crucial. Treatment modalities include volume optimization, improvement of myocardial contractility, restoration of perfusion pressure as well as mechanical circulatory support [11].

It is unexpected for hypoxemia to persist after intubation and the likely causes should be ruled out in a logical fashion. These causes are classified into equipment-related problems, progression of the underlying disease, the onset of a new problem and the side effects of interventions, procedures and medications (Table 1) [12, 13]. The airway, breathing, circulation, disability, exposure (ABCDE) approach recommended in clinical emergencies [14] should be adopted to confirm the diagnosis. Airway patency is confirmed by inspecting the ETT and breathing circuit to rule out blockages and leaks, and ventilator settings should be optimized. Auscultation findings can confirm endobronchial or esophageal intubation, and suggest presence of bronchospasm, pulmonary edema, consolidative changes or pneumothorax. The hemodynamic parameters should be checked and hypotension corrected. Patient exposure can reveal further clues to assist with the diagnosis, such as the presence of rashes in anaphylaxis. Lastly, a review of drugs and doses administered should be done. Additional investigations may be required to achieve a definitive diagnosis. A CXR can be obtained to confirm ETT placement, and rule out pneumothoraxes, atelectasis or lung collapse, and pneumonia. The placement of ETT can be further confirmed by repeat laryngoscopy to verify that the ETT is seen passing through the vocal cords. A CT pulmonary angiogram can be done to rule out pulmonary emboli, especially if the clinical suspicion is high.

In conclusion, this is a rare complication of Stanford type A aortic dissection, where the patient developed severe persistent hypoxemia despite intubation and ventilatory support due to a dissection that extended to the RCA and resulted in RVF. Medical practitioners should consider this as a potential etiology when next faced with a similar patient. Nevertheless, the rarity of such a presentation demands that common causes of hypoxemia are excluded first. 


\section{Learning points}

Aortic dissection can have diverse presenting symptoms and complications as a result of the location and extent of dissection flap involved. RVF is a rare possible complication as a result of dissection flap extending to RCA causing right heart infarction and akinesia. Additionally, RVF is not easily diagnosed clinically due to its non-specific features, and persistent profound hypoxemia is a possible presentation although this is rarely described. The outcomes of such patients are dependent on swift recognition of both the primary condition of aortic dissection and the presence of RVF, to allow institution of timely interventions which have been shown to have a survival benefit. Lastly, the rarity of such a presentation demands that common causes of hypoxemia are excluded first.

\section{Acknowledgments}

None to declare.

\section{Financial Disclosure}

None to declare.

\section{Conflict of Interest}

None to declare.

\section{Informed Consent}

Obtained from the patient's next-of-kin.

\section{Author Contributions}

Shi Hui Tan wrote the manuscript and is the corresponding author. Cheryl Chun Lei Tan contributed to the writing of the manuscript. Yi Lin Lee conceptualized the idea of writing the manuscript and contributed to the writing of the manuscript.

\section{Data Availability}

The authors declare that data supporting the findings of this study are available within the article.

\section{References}

1. Isselbacher EM. Epidemiology of thoracic aortic aneu- rysms, aortic dissection, intramural hematoma, and penetrating atherosclerotic ulcers. In: Eagle KA, Baliga RR, Isselbacher EM, et al. eds.; Aortic Dissection and Related Syndromes. New York: Springer, 2007: p. 3-15.

2. Melvinsdottir IH, Lund SH, Agnarsson BA, Sigvaldason K, Gudbjartsson T, Geirsson A. The incidence and mortality of acute thoracic aortic dissection: results from a whole nation study. Eur J Cardiothorac Surg. 2016;50(6):11111117.

3. Evangelista A, Isselbacher EM, Bossone E, Gleason TG, Eusanio MD, Sechtem U, Ehrlich MP, et al. Insights from the international registry of acute aortic dissection: a 20year experience of collaborative clinical research. Circulation. 2018;137(17):1846-1860.

4. Januzzi JL, Eagle KA, Cooper JV, Fang J, Sechtem U, Myrmel T, Evangelista A, et al. Acute aortic dissection presenting with congestive heart failure: results from the International Registry of Acute Aortic Dissection. J Am Coll Cardiol. 2005;46(4):733-735.

5. Mashar RP, Birla RP, Waterworth P. Right ventricular failure following acute Type A aortic dissection: an alternative strategy. World Journal of Cardiovascular Surgery. 2019;9(3):35-39.

6. Szczechowicz M, Weymann A, Karck M, Szabo G. Right ventricular failure following acute Type A aortic dissection successfully treated with ECMO: report of two cases. J Clin Case Rep. 2016:906.

7. Margulescu AD, Wheeler R, Leong FT. Hypoxia exacerbated by mechanical ventilation: when the trap door opens in severe right ventricular failure. Heart. 2013;99(14):1066.

8. Amsel BJ, Rodrigus I, De Paep R, De Raedt H, Moulijn AC. Right-to-left flow through a patent foramen ovale in acute right ventricular infarction. Two case reports and a proposal for management. Chest. 1995;108(5):14681471.

9. Ibrahim BS. Right ventricular failure. E-Journal of Cardiology Practice. 2016;14(32).

10. Albakri A. Right heart failure: A review of clinical status and meta-analysis of diagnosis and clinical management methods. Int Med Care. 2018.

11. Arrigo M, Huber LC, Winnik S, Mikulicic F, Guidetti F, Frank M, Flammer AJ, et al. Right ventricular failure: pathophysiology, diagnosis and treatment. Card Fail Rev. 2019;5(3):140-146.

12. Glauser FL, Polatty RC, Sessler CN. Worsening oxygenation in the mechanically ventilated patient. Causes, mechanisms, and early detection. Am Rev Respir Dis. 1988;138(2):458-465.

13. Keith RL, Pierson DJ. Complications of mechanical ventilation. A bedside approach. Clin Chest Med. 1996;17(3):439-451.

14. Thim T, Krarup NH, Grove EL, Rohde CV, Lofgren B. Initial assessment and treatment with the Airway, Breathing, Circulation, Disability, Exposure (ABCDE) approach. Int J Gen Med. 2012;5:117-121. 